\title{
Research on the reform of Web front-end courses based on Internet + Mode
}

\author{
Yingan Shao ${ }^{a}$, Guichun Li and Gang Liu \\ School of Computer Science, Baicheng Normal University, Baicheng 137000, China; \\ ashaoyingan@sina.com
}

Keywords:Web3.0, Front-end Course,Progressive Online Project Case.

\begin{abstract}
This paper analyzes and researches deeply the main problems existing in the teaching process of Web front-end courses based on the Internet mode.It also puts forward the theory knowledge system of Web 3.0 front-end course and the core idea of creating progressive online project case, thus changing the students' traditional learning methods and making the students change from theoretical memorizing learning to practical learning.And gradually reform the computer room offline teaching mode and examination mode, ensure that students master the latest related theories, technologies and tools of Web front-end courses as soon as possible, so that students can adapt to the needs of enterprises for Web front-end talents.
\end{abstract}

\section{A Review of domestic and Foreign Research status}

\subsection{Relevant research on the theoretical knowledge system of Web front-end courses.}

In recent years, based on Internet + mode, around the Web front-end curriculum theory knowledge system reform and exploration of related research, from the current knowledge of literature, mainly focus on the teaching content, teaching mode and study on the corresponding talent training, etc.Such as The teaching reform and practice of Web front-end development technology course by Jiuliang Chu. Computer education,2014(14); Liqing Zeng's Research on the front-end development framework of web application for web 2.0.Technology and innovation,2015(22) ; Research on the exploration and talent cultivation of Web front-end technology course content by Song Yang. information and computer (theory), 2016(11); Reflection on WEB programming course teaching by Liu Ni. Journal of guiyang college (natural science edition),2017(04) and other Scholars discuss the importance and necessity of the reform of the theoretical knowledge system of Web front-end courses from different perspectives.

\subsection{Related research on project case teaching.}

Case teaching as a kind of teaching mode is widely applied in the system of higher education, especially those who have strong course requires students to practice is especially important in the teaching, at present scholars mainly focus on aspects such as case teaching, case selection and evaluation system for related research.Such as Jinghua Sun's Application of case teaching method in Web programming teaching. Chinese science and technology information,2008(20); The practice and exploration of the reform of college classroom teaching mode by case teaching by Huarong Wang. Chinese university teaching,2011(04);Application of extensibility case in the teaching of Web application development technology by Huiling Guo. Journal of China environmental management cadre college,2013,23(01);Case driven Web technology and application teaching reform exploration by Dai Tao.China science and education innovation guide,2014(08).Web programming case driven teaching research by Dongmei Guo.Journal of anqing normal university (natural science edition),2016,22(01); and other Scholars discussed the concrete application of case teaching in teaching Web front-end, and also made the relevant beneficial research and exploration in the Web front-end course, Although the project case method is used for teaching, but most of the project cases are just off-line, can only be run in the computer room, lab, not directly to online (such as student write page, not able to run on the Server background, students' learning experience poor, low sense of achievement), and even if some schools have created a project case library website, just provide 
relevant resources, project case practical value play not to come out, resulting in the disconnection between the theory and the practical application.

\section{Research objectives, main contents, important viewpoints and innovations of this thesis}

\subsection{Research objectives of this thesis.}

Build Web front-end curriculum theory knowledge system, let the students master the Web front-end development technology comprehensively, and expand the case teaching method and introducing gradual online project case, thus causes the student to learn and play, so that students in a project case, according to the actual business requirements in terms of object-oriented programming and modular programming, thus improving students' comprehensive ability to use knowledge, project development experience, for later use mainstream Web front-end framework consolidate solid foundation for enterprise development, help to improve students programming ability and practice level.

\subsection{The main contents of this thesis.}

1 Establishment of theoretical knowledge system of Web front-end courses.

1.1 the basic knowledge system of Web front-end course theory is unreasonable and the teaching content is old.

At present, the Web front-end technology has undergone tremendous changes, which are mainly divided into three typical stages: the first stage is that Web 1.0 mainly adopts HTML and CSS as the mainstream technology. The second stage is Web 2.0, which mainly uses JavaScript, DOM and asynchronous data to request Ajax applications as the mainstream technology.The third stage is the Web 3.0 which mainly adopts HTML + CSS3 technology application for the mainstream, while most colleges and universities related Web front-end theory course knowledge system Settings, can't keep up with the Web front-end technology the pace of change, the relevant course content is too old, most only stays in the stage of Web2.0, more serious front only speak Web1.0, the vast majority of colleges and universities the corresponding Web front-end curriculum update speed slow.

However, most of the contents of the teaching materials are listed as knowledge points, and the theoretical knowledge is not penetrated into the specific application cases. It is very difficult for students to master the knowledge points learned in the actual development of how to carry out the specific application, therefore,Therefore, it is very important for Web front-end curriculum theoretical knowledge system to be formulated as soon as possible and to introduce project cases with practical application background.

1.2 Web front-end development tools selection.

At present, most of the design tools used in Web front-end courses in colleges and universities still stay on the visual design tools such as DW. The development and design of all codes are mostly dependent on the design tools. At ordinary times, students will only use the mouse to generate pages.The result is that students will only use the design tool, leave the design tool, not write the full page code, and the industry mainstream will introduce fewer editors such as WebStorm or VsCode, so,As a direct result, students are out of touch with enterprise development tools after graduation, and consider the theoretical knowledge system of Web front-end courses. Reference to the latest development tools, so that you can connect with the industry, and use the latest Web front-end technology and mainstream frameworks.

2 progressive online project case.

2.1 status and dilemmas of project cases.

At present, there is a general lack of practical application scenes in Web front-end course projects in colleges and universities. The typical cases with strong functionality, good user experience, beautiful interface, and students' accumulated project development experience are few.The reason for this bottleneck in docking with enterprise-level development is mainly caused by these four kinds of reasons. The first reason is that the Web front-end theoretical knowledge is too much, particularly trivial and messy, and the technical details have their own history of development.If the same 
knowledge point is different for different browsers, students are difficult to learn and have a lot of memorization, so it is difficult to master the practical application of the theoretical knowledge.

Second such reason is the industry on the front-end technology update speed, platform tools evolved rapidly, various versions of the front-end technology and emerge in an endless stream between each version on grammar and usage of the difference is too large, not compatible, caused students' time cost is too high, the gradient learning is too large, often hasn't learned to use this function, the next version of it cancel this usage. Third reasons in all changes, the biggest change is the Web framework in front of the mainstream, but the application of these frameworks, there must be certain before continued on the basis of practical experience and profound, can use these framework for enterprise development. Fourth kinds of reasons, in Among all the links, teachers in colleges and universities must be familiar with the mainstream development technology, relevant theories and development tools of the industry, and require a relatively high level of theory and practice, which is the core and hub of the teaching process. The goal of introducing progressive online project cases is to reduce the difficulty and threshold of the main enterprise-level development framework for students.

2.2 Construction of progressive online project cases.

It is a very difficult project how to drop the case of progressive online project.The project case construction of this subject mainly starts from five aspects. The first aspect is to integrate the online case video resources, draw lessons from the new teaching mode, such as "MOOC", "flip class", "distance education", "micro class" and "hybrid teaching", according to the characteristics of this course.Collect high-quality video course resources suitable for students' knowledge level, improve interactive teaching experience and provide personalized learning services.In the second aspect, the project practice textbook is selected or modified, and the project and task are decomposed to form a progressive online project case base.The third aspect, from the teacher's own team, Start, simulate or develop real project development, and transform your actual development project into a progressive online project case. The fourth main plane, from the relevant school and enterprise cooperation units or internship units to obtain enterprise-level development projects, digestion and absorption into progressive online project cases.The fifth aspect, the construction of progressive project case teaching database server.The goal of progressive online project case construction is to enable students to participate in many complete and real project development in the course of Web front-end course, so as to enhance the students' actual development ability.

\subsection{The important viewpoints of this thesis.}

1. The concept of Web front-end curriculum theory knowledge system.

First, the theoretical knowledge system of Web 3.0 front-end course is comprehensively consolidated, which is composed of three parts. The first part of HTML mainly includes the technical points of tag semantics and syntax commonly used in HTML4.0.1, and the new tags and new features of HTML5.The second part of CSS mainly includes CSS syntax, selector, list table, font border style, background, positioning, floating.Box model CSS3 adds selectors and adds Flex elastic layout attributes with 2D / 3D conversion and transition web fonts and animation properties.Part III JavaScript mainly includes data type DOM / BOM operation, various events, client-side storage technology, multimedia image programming JS special effects and ECMAScript 6 new JavaScript object, object oriented, prototype, scope chain, The method of calling function and the direction of this, the data structure of regular expression set and Map and the design pattern of JS make students master the basic skills of Web development. Second, introduce the current mainstream framework and development and testing tools of the Web front end, and carry out the corresponding framework project development. Three, master the technology of setting up the backstage at the end of the server.

2. The concept of progressive online project case design.

The main contents are as follows: First, Progressive online project cases are compiled by "project-task-driven" teaching method, and the important knowledge points are integrated into the project tasks, so that students can grasp the technical key points of Web front-end development flexibly. Second, all project case code is divided into two versions, one version is offline project case, the other version is online project case. Thirdly, the teaching design adopts the teaching mode of "the 
theory of task demand to support the gradual realization of online project case", thus arousing the students' interest and enthusiasm in learning, and experiencing the core idea that they have learned.

3.The Innovations of this thesis.

The innovation of this subject is as follows: First, the theoretical knowledge system of Web 3.0 front-end curriculum is put forward: according to the division of theoretical knowledge system of Web front-end curriculum, the core theoretical curriculum content of each stage is decomposed and refined.According to the order of difficulty and difficulty of knowledge points, this paper combs the important knowledge points and teaching difficulties and formulates the training plan and teaching outline.Secondly, the paper puts forward the case of the progressive online project: creating and combining the specific application requirement background, according to the way of "explaining the requirement analysis practice and the server side interaction" according to the theory.For progressive online project case development and upload to The designated teaching server can be published and displayed to enable students to achieve the trinity of experiential learning from theory to practice to application and truly to the practical use of learning.

\subsection{Research ideas, research methods and implementation steps of this paper.}

(I) Research ideas and implementation steps

The first step: through collecting related domestic and foreign literature, combing and analyzing and researching.The second step: to develop the theoretical knowledge system of Web 3.0 front-end courses to meet the teaching requirements of applied undergraduate colleges. The third step: collect and organize and write progressive online project cases, truly create a better teaching model for students, and build the project case server platform.

(II) Research Methodology

Document analysis: through extensive collection, research on relevant literature on the subject, drawing on advanced ideas, and selectively learning from the current educational ideas for further in-depth research.

Case analysis method: based on the Internet mode, integrating online and offline resources, combining with its own Web front-end course specialty characteristics, build a case server platform suitable for this specialty project.

Experience summary method: in a typical teaching link, we should track and respond to other students' teaching effect in time, integrate practice and theory, and constantly revise and renew teaching plan and teaching means.

\section{Summary}

This paper expounds in detail the main problems existing in the teaching process of Web front-end courses, puts forward emphatically the theoretical knowledge system of Web 3.0 front-end courses, combs the key points and difficulties of integrating the theoretical knowledge systems and formulates the corresponding training schemes and teaching syllabuses.

It also puts forward the case of the progressive online project, which enables the students to achieve from theory to practice to application, and lays a solid foundation for the training of qualified Web back-end development engineers.

\section{References}

[1]. Jiuliang Chu. The teaching reform and practice of Web front-end development technology course. Computer education.Vol.14(2014) No.7, p.12-15.

[2]. Liqing Zeng. Research on the front-end development framework of web application for web 2.0.Technology and innovation.Vol.22(2015) No.11, p.16-17.

[3]. Song Yang. Research on the exploration and talent cultivation of Web front-end technology course content.Information and Computer (theory).Vol.11(2016) No.6, p.237-238. 
[4]. Liu Ni.Reflection on WEB programming course teaching.Journal of guiyang college (natural science edition).Vol.4(2017) No.12, p.27-28+39.

[5]. Jinghua Sun. Application of case teaching method in Web programming teaching. Chinese science and technology information;Vol.20(2008) No.10, p.290-291. 